\title{
EFECTO DE LA INTERFERENCIA PARCIAL EN LA DEPREDACIÓN NATURAL SOBRE UNA COMUNIDAD DE ACRÍDIDOS EN UN PASTIZAL DEL DESIERTO CHIHUAHUENSE
}

\author{
Eduardo Rivera García ${ }^{\mathbf{1}}$ y Zenón CaNO-SANTANA ${ }^{\mathbf{2}}$ \\ ${ }^{1}$ Instituto de Ecología, A. C. (INECOL), Centro Regional Durango. Apdo. Postal 632. Km 5 carretera \\ a Mazatlán, Esq. Blvd. Los Remedios. Los Remedios, Durango, Dgo., MÉXICO. \\ e-mail: eduardo.rivera@inecol.edu.mx \\ 2 Departamento de Ecología y Recursos Naturales, Facultad de Ciencias, Universidad Nacional \\ Autónoma de México. Ciudad Universitaria, Coyoacán. 04510 MÉXICO, D.F. \\ e-mail: zcs@hp.fciencias.unam.mx
}
Rivera García, E. y Z. Cano-Santana. 2009. Efecto de la interferencia parcial en la depredación natural sobre una comunidad de acrídidos en un pastizal del Desierto Chihuahuense. Acta Zoológica Mexicana (n. s.), 25(2): 345-357.

RESUMEN. Durante el verano de 1998, se analizó el efecto de interferencia parcial por medio de la remoción de depredadores, en una comunidad compuesta de ocho especies de acrídidos durante un brote masivo poblacional de una de ellas, Boopedon nubilum (Say), en una estepa subarbustiva de zacate toboso en el Desierto Chihuahuense. El diseño consistió de cuatro bloques con un tratamiento (depredadores disminuidos parcialmente) y un control. Las especies de depredadores registradas incluyeron a cinco mamíferos, cinco reptiles, dos anfibios, 20 aves, seis insectos diurnos, seis arañas y cuatro artrópodos depredadores de hábitos nocturnos. Los depredadores vertebrados importantes fueron las aves. La presencia de depredadores vertebrados inhibió la actividad de los depredadores invertebrados. No hay efecto significativo entre las fechas y tratamiento, sobre las densidades de acrídidos. El efecto de la depredación en la población de B. nubilum, modificó la composición de especies de la comunidad de acrídidos y las plantas usadas por los acrídidos para forrajeo y/o protección. Las especies graminícolas mostraron una estrategia de escape que incluye saltar entre los zacates, macollos o al suelo desnudo, disminuye el riesgo de captura, de acuerdo a su coloración y de la posición final que adoptan para evitar ser detectados. La ausencia de un efecto significativo de la depredación se atribuye al bajo impacto de la perturbación y al corto tiempo de respuesta, así como a un probable fenómeno de saciar su apetito por la abundancia de alimento debido al brote masivo de $B$. nubilum. Sin embargo se detectaron cambios en la composición de la comunidad de especies de acrídidos. Las parcelas de control y tratamiento mostraron una tendencia negativa sobre los registros de densidad de B. nubilum y un efecto natural de la depredación. Sugerimos que para este tipo de experimentos se deberá aplicar el disturbio durante un periodo de tiempo mayor y con una remoción total o casi total de los depredadores, así como monitorear las densidades de la siguiente generación de acrídidos, para poder determinar el efecto retardado de la depredación en la siguiente generación.

Palabras clave: interferencia, depredación, Desierto Chihuahuense, comunidad de acrídidos, chapulines.

Recibido: 8/07/2008; aceptado: 11/02/2009. 
Rivera García, E. \& Z. Cano-Santana. 2009. Effect of partial interference on natural predation of a grassland grasshopper community from the Chihuahuan Desert. Acta Zoológica Mexicana (n. s.), 25(2): 345-357.

ABSTRACT. Perturbation on natural predation in a driest year (1988) on eight grasshopper species assembly during an outbreak of one of them (Boopedon nubilum) in a toboso arid grassland in Chihuahuan Desert was analyzed. We used a four blocks trial with a treatment (predators' decreased) and a control. Predators recorded were five mammals, five reptiles, two amphibians, 20 birds, six diurnal Insects, six spiders and four nocturnal arthropods. The vertebrate predators presence (principally raptors) inhibited invertebrate predators action, the most important predators were birds. Results showed no significative effect of date and treatment on grasshopper density (pooled data). The effect of predation on the most abundant grasshopper species (B. nubilum) was observed, changing the species composition of the assembly and the use of plant stratification by grasshoppers. The graminicole species showed an escape strategy of jumping between grasses, tussocks, bunches or to bare soil, or only keep down into the grass, using this strategy, vulnerability (detection risk by predators) depends on their coloration and final position adopted to avoid predators. The short time effect results were due by short time response in treatment add to the predation satiation phenomena by over offer of meal in this specific patch by the outbreak grasshopper species. Where control and treatment showed clearly negative tendency on the population records of $B$. nubilum and a natural effect of predation, but our statistical analysis showed no significative differences, suggesting it is more adequate to do this kind of trials, considering a larger remotion of predators and monitoring and evaluating on next generation of grasshopper species.

Key words: interference, predation, Chihuahuan Desert, grasshopper community.

\section{INTRODUCCIÓN}

Existe gran cantidad de trabajos que demuestran que la depredación puede tener una influencia significativa en las poblaciones, en la estructura de la comunidad, en la dinámica de las redes tróficas, especialmente en las especies de herbívoros dominantes (Hairston et al. 1960, Paine 1966, Hunter \& Price 1992, Hairston \& Hairston 1997), incluyendo a los insectos (Marquis \& Whelan 1994) entre los que se cuentan los acrídidos (Beckerman et al. 1997, Schmitz et al. 1997, Schmitz 1998). Por otra parte, en los ecosistemas de pastizal, los acrídidos son los herbívoros dominantes (Odum et al. 1962, Van Hook 1971, Mitchel \& Pfadt 1974, Risser et al. 1981), mostrando una intrincada red de interacciones tróficas, entre insectos herbívoros, parásitos, parasitoides y depredadores vertebrados e invertebrados (Dempster 1963, Greathead 1963, Lavigne \& Pfadt 1966, Rees 1973, Polis 1991).

Aunque los parasitoides constituyen el principal factor de mortalidad para insectos fitófagos, los depredadores son un factor extra que puede ser importante en la reducción y regulación de la densidad de población de acrídidos (Stower \& Greathead 1969, Joern \& Rudd 1982, Joern 1986, Joern \& Gaines 1990, Belovsky \& Slade 1993), en particular las aves (Bock et al. 1992). Los depredadores generalistas pueden proporcionar una fuerte presión de selección en el mantenimiento de las poblaciones y en la evolución de insectos herbívoros, también constituyen un factor importante que puede modificar la composición de la comunidad (Bernays \& 
Graham 1988, Kaspari \& Joern 1993, Rothley et al. 1997, Rosenheim 1998, Pitt 1999, Rota \& Wagner 2006), en respuesta a esta presión evolutiva, muchas especies de acrídidos, han adquirido adaptaciones morfológicas, coloraciones y conductas para evitar ser detectados (Foreman \& Appelqvist 1998, Beckerman et al. 1997, Ovadia \& Schmitz 2004, Schmitz et al. 2000, Civantos et al. 2004).

Por lo anterior es importante definir el papel de los depredadores en la dinámica de población de los acrídidos y su efecto en la proporción de especies que componen las comunidades de herbívoros. Este fenómeno es especialmente interesante cuando ocurren brotes masivos en la población de alguna de las especies de tal ensamble.

Boopedon nubilum (Say) es una especie graminícola que muestra un marcado dimorfismo sexual, el cuerpo de los machos (24-34 mm), es siempre de color negro, las hembras (33-52 $\mathrm{mm}$ ) presentan coloraciones que van desde el color amarillo paja, café pálido, café obscuro a negro obscuro en su totalidad, en la reserva de la biosfera Mapimí (RBM), es frecuente observar brotes masivos de esta especie (Rivera 2006).

En este trabajo, mostramos los resultados relacionados con varios aspectos de la interferencia en la depredación natural (disturbio), durante un brote natural de la población de B. nubilum. Los objetivos de este trabajo fueron: (1) Determinar cuál es el efecto a corto plazo, de la perturbación en la depredación a nivel de comunidad y de la especie que mostró un brote masivo de su población; (2) Determinar qué clase de depredadores de acrídidos son importantes durante un brote masivo poblacional bajo condiciones de disturbio; y (3) determinar qué mecanismos usan los acrídidos para evitar su ataque.

\section{MATERIAL Y MÉTODOS}

Área de estudio. Este trabajo fue realizado en una estepa sub-arbustiva dominada o codominada por zacate toboso Hilaria mutica (Buckl.), Benth., Prosopis glandulosa Torr. y Larrea tridentata (C.C.) Cov., ubicada a 1.5 kilómetros al norte de la estación del INECOL "Laboratorio del Desierto," en la Reserva de la Biosfera Mapimí, situada en el área del Bolsón de Mapimí, en la parte mexicana del Desierto Chihuahuense (polígono irregular ubicado entre $26^{\circ} 10^{\prime}$ y $27^{\circ} 00^{\prime}$ norte, y $103^{\circ} 25^{\prime}$ y $104^{\circ} 05^{\prime}$ oeste; con registros de altitud entre 1050 y $1350 \mathrm{~m}$ ). El área es dominada por terrenos llanos con arbustos, pastizales áridos, áreas desnudas de vegetación y colinas discontinuas. El clima es cálido y seco, con temperatura media mensual que oscila entre 12 y $28^{\circ} \mathrm{C}$, la precipitación anual es $263 \mathrm{~mm}$ y ocurre principalmente en verano, la evaporación anual es de alrededor de 2,796 mm (Cornet 1988).

Experimento. Se llevó a cabo en el verano de 1998. Antes, durante y después de éste, como complemento se realizaron actividades para determinar la identidad de todos los depredadores en los sitios de estudio, mediante colecta directa, registro por observación y búsqueda de restos de chapulines en heces. La composición y cobertura de la vegetación fue evaluada por medio de cinco líneas de interceptación 
tipo Canfield, de $20 \mathrm{~m}$ de largo en cada parcela experimental (Brower \& Zar 1980). Las parcelas experimentales fueron instaladas a mediados de septiembre y fueron utilizadas entre el 20 de septiembre y el 7 de octubre. El diseño experimental consistió de cuatro bloques de dos parcelas de 36 × $36 \mathrm{~m}$ con un tratamiento (depredadores reducidos) y un control (con la presencia natural de depredadores), con aproximadamente 20-25 m entre ellas, cada bloque separado por al menos 500 $\mathrm{m}$. El experimento se llevó a cabo dentro de un área ocupada por la especie responsable del brote poblacional (B. nubilum).

En las parcelas sujetas a disminución de depredadores, se usó un "espantapájaros" como fuente de interferencia para alejar a las aves. Este espantapájaros consistió en una bola de esponja con dos cintas de color rojo de $1.20 \mathrm{~m}$ de largo, la cual era arrojada a las aves. Este método fue utilizado durante $4 \mathrm{~h}$ en cada parcela a diferentes horas del día (dos horas por la mañana y dos horas por la tarde). Las aves alejadas (todas), se registraron como "removidas", durante el tiempo entre los conteos de acrídidos. Cada día, los depredadores como lagartijas, coyotes, etc., fueron removidos o alejados por interferencia directa (dos horas por la mañana y el mismo tiempo por la tarde), en tanto que otros depredadores más pequeños fueron capturados y removidos usando 10 trampas Sherman y 20 pitfall $(20 \mathrm{~cm}$ de alto y 12 $\mathrm{cm}$ de diámetro sin preservador) por parcela, las cuales fueron revisadas dos veces al día (en la mañana y en la tarde), entre los conteos de chapulines en los periodos comprendidos entre los días quinto y décimo $\left(\mathrm{T}_{5-10}\right)$ y entre los días décimo y decimo quinto $\left(\mathrm{T}_{10-15}\right)$ contados a partir del primer censo de chapulines $\left(\mathrm{t}_{0}\right)$.

Todos los depredadores considerados como "removidos" fueron contados, determinados y liberados a la mayor distancia posible del área del trabajo (al menos $200 \mathrm{~m}$ ). Los acrídidos fueron contados visualmente, usando cinco bandas/transecto de $1 \times 30 \mathrm{~m}$, ubicadas en forma paralela a una distancia de $5 \mathrm{~m}$ entre ellas, cada cinco días, $\left(t_{0}, t_{+5}, t_{+10}, \mathrm{y} \mathrm{t}_{+15}\right)$, registrando la abundancia, la forma de vida (Uvarov 1977) y el substrato usado (Rivera 1988, 1992, 2006) y calculando la densidad de cada especie de acrídido (no. de individuos $/ \mathrm{m}^{2}$ ) dentro de cada parcela (tratamiento y control). La identificación de acrídidos fue realizada usando las claves de Rivera (1986) y Richman et al. (1993). B. nubilum emergió alrededor del 20 de agosto de 1998, antes del inicio del trabajo en el terreno, y continuó activo hasta principios de noviembre.

Los datos promedio de densidad de chapulines fueron analizados por medio de un ANDEVA (Steel \& Torrie 1960, 1976, Zar 1996), sin tomar en cuenta los registros a $\mathrm{t}_{0}$ debido a que la población de $B$. nubilum se encontraba en estado preadulto.

El efecto de la depredación en la composición de especies de la comunidad de acrídidos fue analizado por medio de una serie de pruebas de Chi cuadrada aplicadas a los registros agrupados por forma de vida (Rivera 2006), considerando o no los registros de $B$. nubilum y por último los mismos registros agrupados pero tomando en cuenta por separado los registros del control y los del tratamiento. 


\section{RESULTADOS}

La cobertura vegetal en todas las parcelas fue estimada en alrededor de $60 \%$, sin diferencias aparentes entre ellas y siempre dominada por gramíneas acompañadas de hierbas y arbustos, sin embargo se observó una cobertura muy baja de plantas anuales. Se registraron ocho especies de acrídidos: Boopedon nubilum (Say), Bootettix argentatus Bruner, Brachystola magna Scudder, Hesperotettix viridis (Thomas), Ligurotettix planum (Bruner), Opeia obscura (Thomas), Paropomala virgata (Bruner) y Trimerotropis pallidipennis (Burmeister). La densidad más alta registrada correspondió a la forma de vida graminícola y las más bajas a terrícolas y arborícolas (Cuadro 1).

Cuadro 1. Abundancia registrada de ocho especies de acrídidos en pastizales de la Reserva de la Biosfera Mapimí en el verano de 1998. Especies encontradas de chapulines de acuerdo a sus formas de vida: Arborícolas: BOAR Bootettix argentatus; LIPL Ligurotettix planum;

HEVI Hesperotettix viridis; BRMA Brachystola magna Graminicolas: BONU Boopedon nubilum;

OPOB Opeia obscura; PAVI Paropomala virgata terricola: TRPA Trimerotropis pallidipennis.

$\mathrm{t}_{\mathrm{n}}$ censo de chapulines al tiempo $\mathrm{n}\left(0=\right.$ inicial; $5=$ al $5^{\circ}$ día; $10=$ al $10^{\circ}$ día; $15=$ al $15^{\circ}$ día $)$.

$\mathrm{C}=$ abundancia en parcelas control al tiempo $\left(\mathrm{t}_{\mathrm{n}}\right)$,

$\mathrm{T}=$ abundancia en parcelas con interferencia al tiempo (tn).

\begin{tabular}{lrrrrrrrr}
\hline & & $\mathbf{t}_{\mathbf{0}}$ & & $\mathbf{t}_{\mathbf{5}}$ & \multicolumn{2}{c}{$\mathbf{t}_{\mathbf{1 0}}$} & \multicolumn{2}{c}{$\mathbf{t}_{\mathbf{1 5}}$} \\
\hline SPP & $\mathbf{C}$ & $\mathbf{T}$ & $\mathbf{C}$ & $\mathbf{T}$ & $\mathbf{C}$ & $\mathbf{T}$ & $\mathbf{C}$ & $\mathbf{T}$ \\
\hline BONU & 384 & 638 & 320 & 532 & 149 & 378 & 213 & 310 \\
BOAR & 8 & 8 & 8 & 8 & 11 & 8 & 0 & 1 \\
BRMA & 0 & 0 & 0 & 0 & 0 & 0 & 2 & 5 \\
LIPL & 4 & 5 & 4 & 5 & 6 & 7 & 1 & 2 \\
OPOB & 8 & 12 & 10 & 16 & 4 & 14 & 40 & 47 \\
PAVI & 49 & 22 & 49 & 22 & 34 & 17 & 0 & 1 \\
TRPA & 12 & 15 & 16 & 17 & 20 & 14 & 18 & 21 \\
HEVI & 0 & 0 & 0 & 0 & 0 & 0 & 0 & 1 \\
\hline TOTAL & 465 & 700 & 407 & 600 & 224 & 438 & 274 & 388 \\
\hline
\end{tabular}

Al inicio del trabajo (14/sept/98), la población de B. nubilum se encontraba en estadios ninfales, con una densidad media de $35.81 \pm 35.20$ ind. $/ \mathrm{m}^{2}$ d. e. $(\mathrm{n}=70)$ con un máximo de 79 ind. $/ \mathrm{m}^{2}$ y un mínimo de 0 ind. $/ \mathrm{m}^{2}$, ocupando una área estimada entre 20 y 30 ha.

Aunque la actividad de aves depredadoras se redujo ligeramente en el ( $\left.\mathrm{T}_{10-15}\right)$ en las parcelas de control, las parcelas de tratamiento mostraron una disminución del número de reptiles (Fig. 1) y un incremento para los artrópodos y aves. 


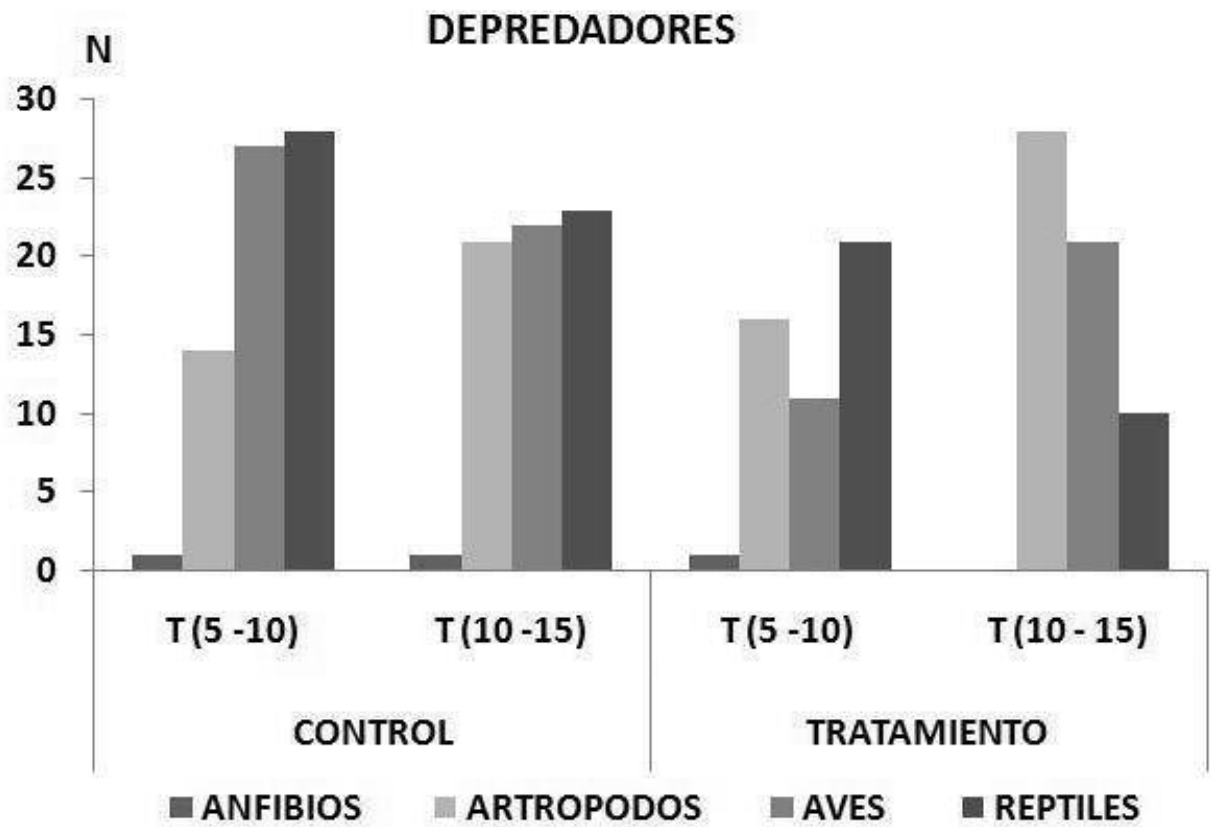

Figura 1. Registro de depredadores "removidos" durante el verano de 1998. N = Número de registros, $\left(\mathrm{T}_{5-10}\right)=$ periodo comprendido entre el quinto y el décimo día, $\left(\mathrm{T}_{10-15}\right)=$ periodo comprendido entre el décimo y el décimo quinto día.

No se encontraron diferencias significativas entre fechas $(\mathrm{F}=7.407 ; \mathrm{g} .1 .=2,10, \mathrm{P}>$ $0.10)$ ni por efecto del tratamiento $(\mathrm{F}=25.909 ; \mathrm{g} .1 .=1,10, \mathrm{P}>0.10)$ debidas a la interferencia de la depredación natural sobre la densidad total de B. nubilum. Se detectó una tendencia a la disminución en la densidad de población (Fig. 2) aunque no se apreciaron cambios en el conjunto de especies de la comunidad pero sí se detectaron cambios apreciables en las proporciones y densidades de especies graminícolas (Fig. 3).

Los resultados de las pruebas de Chi cuadrada aplicadas a los registros de chapulines (Cuadro 3) indican que la principal especie afectada fue B. nubilum, y no hubo efecto sobre las especies con formas de vida terrícola y arborícola. No obstante al excluir los registros de B. nubilum en el análisis sí se detectaron diferencias entre las especies graminícolas. 


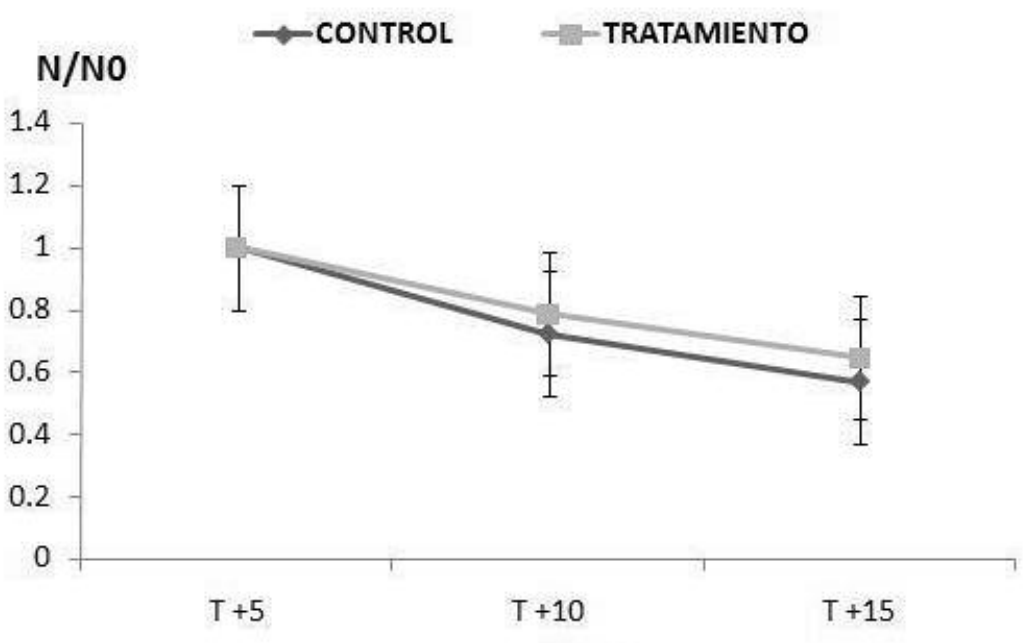

\section{TIEMPO}

Figura 2. Efecto de la depredación natural interferida sobre la densidad de población de Boopedon nubilum, en el verano de 1998. $\mathrm{t}_{+5}=$ censo al quinto día, $\mathrm{t}_{+10}=$ censo al décimo día, $\mathrm{t}_{+15}=$ censo al décimo quinto día, $\mathrm{N} / \mathrm{N}_{0}=$ Densidad al tiempo $\mathrm{T}$, dividido entre la densidad al tiempo inicial (cero).

Cuadro 2. Resumen de los resultados de las pruebas de Chi cuadrada aplicadas a los registros de chapulines en el trabajo de interferencia parcial, todas las especies agrupadas, por formas de vida, considerando y no los registros de la especie responsable del brote de población de Boopedon nubilum (BONU), en el tratamiento (con interferencia) y control (sin interferencia).

\begin{tabular}{lrrrrc}
\hline FUENTE & $\mathbf{X}^{\mathbf{2}}$ cal. & d.f. & $\mathbf{X}^{2}$ tab. & $\boldsymbol{\alpha}$ & significancia \\
\hline & 12.109 & 2 & 9.210 & 0.01 & $* * *$ \\
Todas S/BONU & 4.044 & 2 & 4.605 & 0.10 & $\mathrm{~ns}$ \\
Todas C/BONU & 19.600 & 2 & 9.210 & 0.01 & $* * *$ \\
Solo BONU & 1.209 & 2 & 9.210 & 0.10 & $\mathrm{~ns}$ \\
TERRICOLAS & 16.660 & 2 & 13.815 & 0.001 & $* * * *$ \\
GRAMINICOLAS C/BONU & 4.925 & 2 & 4.605 & 0.10 & $\mathrm{~ns}$ \\
GRAMINICOLAS S/BONU & 2.315 & 2 & 4.605 & 0.10 & $\mathrm{~ns}$ \\
ARBORICOLAS & 13.989 & 4 & 9.488 & 0.05 & $* *$ \\
CONTROL C/BONU & 19.022 & 4 & 13.277 & 0.01 & $* * *$ \\
CONTROL S/BONU & 13.905 & 4 & 9.488 & 0.05 & $* *$ \\
TRATAMIENTO C/BONU & 11.718 & 4 & 9.488 & 0.05 & $* *$ \\
TRATAMIENTO S/BONU & & & & & \\
\hline
\end{tabular}



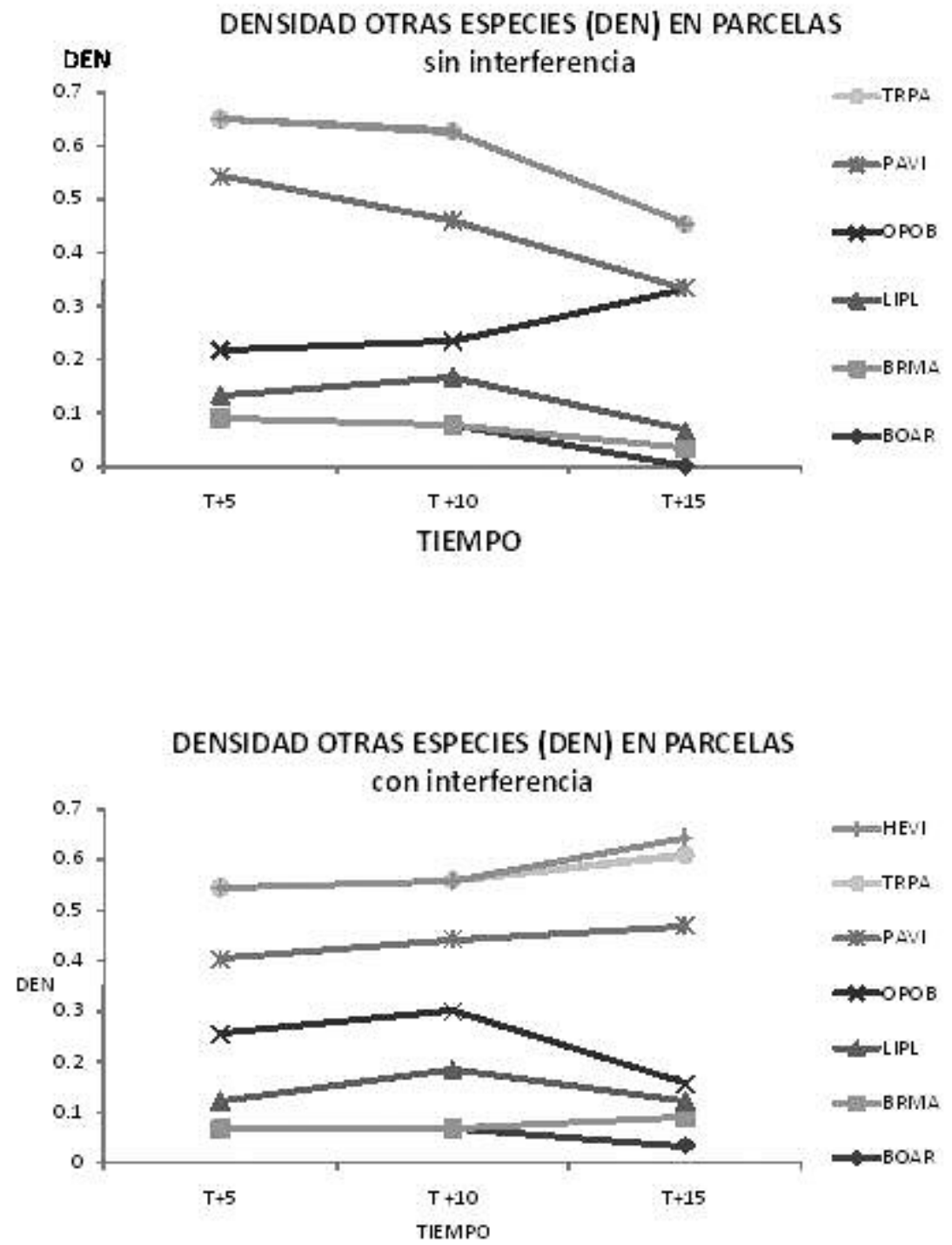

Figura 3. Densidad de las otras especies del ensamble de chapulines registradas durante la depredación natural interferida, en las parcelas de Control (a) y Tratamiento (b), en el verano de 1998. DEN = Número de individuos $/ \mathrm{m}^{2}$; a) Parcela control. b) Parcela tratamiento. HEVI $=$ Hesperotettix viridis $;$ TRPA = Trimerotropis pallidipennis $; \mathrm{PAVI}=$ Paropomala virgata $; \mathrm{OPOB}=$ Opeia obscura;

$\mathrm{LIPL}=$ Ligurotettix planum $; \mathrm{BRMA}=$ Brachystola magna $; \mathrm{BOAR}=$ Bootettix argentatus. 


\section{DISCUSIÓN}

El clima durante 1997 y 1998 fue más seco que la media regional (precipitación 205 y $122 \mathrm{~mm}$, evaporación 2378 y $3285 \mathrm{~mm}$, respectivamente). Esta variabilidad es directa o indirectamente la responsable de las oscilaciones en abundancia de las poblaciones de acrídidos y que muy probablemente detonó el brote masivo de la población de B. nubilum para ese verano (Kemp \& Dennis, 1993, Pitt 1999).

Sólo registramos ocho especies de acrídidos de 12 reportados para este tipo de vegetación por Rivera, (1986), lo cual está estrechamente relacionado con la sequía extrema (Rivera 2006). Los registros de abundancia más alta fueron de acrídidos graminícolas en todo el período de estudio (Cuadro 1).

El efecto del "espantapájaros" fue más notable para las aves rapaces que para las aves insectívoras. De éstas últimas fueron más importantes por su persistencia: Ammodramus savanarum (Gmelin), Dendroica occidentales (Towsend), Lanius ludovicianus Linnaeus, mostrando que se habituaron rápidamente a su efecto no letal. En tanto, en segundo lugar de importancia estuvieron las lagartijas Cnemidophorus inornatus Baird y Sceloporus undulatus (Latreille), (Fig. 1). El efecto de remover a estos depredadores vertebrados benefició a los depredadores artrópodos, debido a que ellos también son consumidos, principalmente por lagartijas.

La perturbación producida por el "espantapájaros" y la remoción de depredadores epígeos mayores no fue muy notorio en los resultados debido al tiempo de duración (solamente $4 / \mathrm{hrs} / \mathrm{día}$ ), por lo que no se encontraron diferencias significativas en el corto plazo durante el ensayo. Aunque los resultados muestran una tendencia negativa de la depredación sobre la densidad de población de los elementos del ensamble de chapulines, en especial sobre B. nubilum (Fig. 2 y Cuadro 2), este efecto puede ser considerado como natural y se suma al posible efecto de los depredadores al saciar su apetito por la abundancia de alimento.

En el campo, las presas enfrentan el riesgo de ser capturadas por múltiples depredadores y los chapulines tienen muchos enemigos naturales (Joern 1986, Belovsky et al. 1990, Bock et al. 1992, Schmitz et al. 1997), por lo que el riesgo que corren los chapulines de acuerdo a la altura a la que se encuentran en la vegetación, explica el hecho de no haber detectado el efecto de la interferencia en la depredación a corto plazo en acrídidos terrícolas y arborícolas $(\mathrm{P}>0.10)$, ni en los registros de todo el ensamble $(\mathrm{P}>0.10)$, cuando excluimos los valores de B. nubilum (ver Cuadro 2). Lo anterior indica que esta especie fue la más atractiva para los depredadores (por sus características morfológicas, color y densidad de población), beneficiando al resto de los elementos del ensamble, lo cual concuerda con los resultados de experimentos realizados por Foreman \& Appelqvist (1998) con aves y con los de Civantos et al. (2004) con lagartijas.

Por otra parte, aunque Wiens \& Rottenberry (1979) sugieren poco impacto de aves sobre los depredadores artrópodos en experimentos de exclusión de aves 
depredadoras de chapulines, sus resultados pueden estar considerando oscilaciones por el efecto de artrópodos depredadores no detectados que quedaron dentro de sus exclusiones. En nuestro caso cuando la interferencia ("espantapájaros" y remoción de depredadores epígeos) produce la reducción del número de depredadores de artrópodos (principalmente lagartijas) durante el brote de población de los chapulines, se disminuye el riesgo de que sean capturados, promoviendo a su vez que la actividad diurna y vespertina de éstos artrópodos depredadores se incremente (Civantos et al. 2004) para aprovechar la abundancia de acrídidos como fuente de alimento (Fig. 1).

Todas las especies graminícolas mostraron tener como estrategia de escape saltar entre las hierbas, los macollos y manojos de hierba o al suelo desnudo (como B. nubilum y $P$. virgata), o protegiéndose en los manojos de hierba (O. obscura), así su vulnerabilidad a la depredación disminuye al distraer ó contrarrestar los esfuerzos de búsqueda del depredador potencial (Staddon \& Gendron 1983, Gendron \& Staddon 1983). Aunque algunas especies tienden a agregarse en áreas densas de vegetación herbácea, donde los acrídidos encuentran los recursos necesarios para su reproducción, supervivencia y para disminución del riesgo de depredación (Hamilton 1971).

En virtud de las densidades de población registradas por B. nubilum, el grupo de los chapulines graminícolas fue el más consumido, aunque se observaron diferencias apreciables en registros de $P$. virgata y de $O$. obscura, esta última especie obtuvo ventajas debido a su estrategia de escape (dejarse caer dentro y abajo de la hierba), disminuyendo el riesgo por su posición y coloración (Foreman \& Appelqvist 1998). B. magna y $H$. viridis, se encontraron cuando la abundancia de graminícolas disminuyó, pero solo la primera especie usó en parte el mismo hábitat que las especies graminícolas, la segunda especie es netamente arborícola (Rivera 2006), ambas se vieron favorecidas debido a la altura de la vegetación en que viven y se desarrollan (Pitt 1999).

De acuerdo con Rivera (2006), B. nubilum es una especie dimórfica, los machos adultos son negros, las hembras comúnmente son de color café obscuro, pero algunas son negras, con una proporción de sexos de 1:1 y la relación de color café-negro en las hembras es 4:1 $(\mathrm{N}=800)$. Esta especie mostró ser una presa atractiva por su tamaño y alto contraste lo que proporciona una buena imagen de búsqueda para los depredadores (Staddon \& Gendron 1983, Gendron \& Staddon 1983); su defensa a la depredación es baja para depredadores vertebrados, por mostrar una alta vulnerabilidad diurna por su color negro (Foreman \& Appelqvist 1998, Civantos et al. 2004). Al incrementar la atención de los depredadores sobre esta especie graminícola, indirectamente disminuye la presión sobre las otras especies graminícolas (Pitt 1999). Esto es razonable debido a que los registros de densidad de B. nubilum obtenidos durante el ensayo fueron diez veces mayores que todas las especies restantes en su conjunto 
No fue posible detectar ningún efecto de la interferencia parcial en la depredación sobre la densidad de chapulines del ensamble a corto plazo, debido a que la disminución de depredadores vertebrados no fue suficiente para evitar su acción sobre la densidad de población de la especie responsable del disparo de población ( $B$. nubilum), ya que esta última por las densidades de población registradas (diez veces mayor que todas las especies en conjunto), sirvió como atracción para los depredadores, disminuyendo el efecto sobre las demás especies, pero sí se detectaron modificaciones en las proporciones y densidades de los acrídidos que componen el ensamble de especies.

También se encontró que el efecto de interferencia ("espantapájaros") sobre las rapaces favoreció a las especies de aves pequeñas y reptiles, quienes mostraron ser los depredadores vertebrados de mayor importancia.

Una limitante para los resultados aquí presentados puede haber sido el poco tiempo invertido para disminuir a los depredadores (4/hrs/día) lo cual, sumado al fenómeno de haber saciado su apetito por la abundancia de alimento proporcionada por el brote masivo de B. nubilum, no permitió detectar el efecto esperado de la interferencia parcial sobre la densidad de acrídidos del ensamble. Estos resultados sugieren que este tipo de diseño experimental debe ser repetido considerando una mayor inversión de tiempo en la disminución de depredadores, un monitoreo más prolongado y evaluar las poblaciones de la siguiente generación de la especie de acrídido que registró el disparo de población.

AGRADECIMIENTOS. Gracias a Juan Francisco Herrera y su familia por invaluable apoyo, ayuda y compañía durante todo el trabajo de campo. Gracias a Alfredo Garza, a Elizabeth Aragón y a Rolando González por su ayuda en la identificación de vertebrados y nombres comunes regionales. Al L. I. Cuauhtémoc Domínguez, por sus valiosos comentarios y revisión del texto. Este estudio fue apoyado parcialmente por el Departamento de Fauna Silvestre del INECOL-CRD, Durango, Dgo., México. Cuentas No. 902-05 y No. 902-08-0028.

\section{LITERATURA CITADA}

Beckerman, A. P., M. Uriarte \& O. J. Schmitz. 1997. Experimental evidence for a behavior-mediated trophic cascade in terrestrial food chain. Proceedings of the. National. Academy of Sciences. 94:10735-10738.

Belovsky, G. F. \& J. B. Slade. 1993. The role of vertebrate and invertebrate predators in a grasshopper community. Oikos. 68:193-201.

Belowsky, G. E., J. B. Slade \& B. A. Stochoff. 1990. Susceptibility to predation for different grasshoppers: An experimental Study. Ecology.71(2):624-634.

Bernays, E. A. \& M. Graham. 1988. On evolution of host specificity in phytophagous arthropods. Ecology. 69:886-892.

Bock, C. E., Bock, J. H. \& Grant, M. C. 1992. Effects of bird predation on grasshopper densities in an Arizona grassland. Ecology. 73: 1706-1717.

Brower, J. E. \& J. H. Zar. 1980. Field and Laboratory Methods for General Ecology. W. M. C. Brown Co. Pub. Dubuque Iowa, E.U. 226 pp. 
Civantos, E., J. Ahnesjö, A. Forsman, J. Martín \& P. López. 2004. Indirect effects of prey coloration on predation risk: pygmy grasshoppers versus lizards. Evolutionary Ecology. 6:201-213.

Cornet, A. 1988. Principales caracteristiques climatiques. Pp. 45-76. In: Montaña C. (ed): Estudio Integrado de los recursos vegetación, suelo y agua en la reserva de la Biosfera de Mapimí. Ambiente Natural y Humano. Pub. 23 Instituto de Ecología, A. C. México, D.F.

Dempster, J. P. 1963. The population dynamics of grasshoppers and Locusts. Biological. Reviews. 38:490-529.

Foreman, A. \& S. Appelqvist. 1998. Visual predators impose correlational selection on prey color pattern and behavior. Behavioral Ecology. 9:409-413.

Gendron, R. P. \& J. E. R. Staddon. 1983. Searching for cryptic prey: the effect of search rate. The American Naturalist.121:172-186.

Greathead, D. J. 1963. A review of insect enemies of Acridoidea (Orthoptera). Transactions of the Royal Entomological Society of London. 114:437-517.

Hairston, N. G., F. E. Smith, \& L. S. Slobodkin. 1960. Community structure, population control, and competition. The American Naturalist. 94:421-425.

Hairston, N. G. Jr., \& N. G. Hairston, Sr. 1997. Does food web complexity eliminate trophic-level dynamics? The American Naturalist. 149: 1001-1007.

Hamilton, W. D. 1971. Geometry for the selfish herd. Journal of Theoretical Biology. 32:295-311.

Hunter, M. D. \& P. W. Price. 1992. Playing chutes and ladders: Heterogeneity and the relative roles of botton-up and top-down forces in natural communities. Ecology. 73:724-732

Joern, A. \& N. T. Rudd. 1982. Impact of predation by the rubber fly Proctacanthus milbertti (Diptera:Asilidae) on grasshopper (Orthoptera:Acrididae) populations. Oecologia. 55:42-46.

Joern, A. 1986. Experimental study of avian predation on coexisting grasshopper populations (Orthoptera: Acrididae) in a sandhills grassland. Oikos. 46:243-249.

Joern, A. \& S. B. Gaines. 1990. Population dynamics and regulation in grasshoppers. Pags. 415-482. In: Chapman, R. F. \& A. Joern (eds). Biology of Grasshoppers. John Wiley \& Sons, New York.

Kaspari, M. E. \& A. Joern. 1993. Prey choice by three insectivorous grassland birds: revaluating opportunism. Oikos. 68:414-430.

Kemp, W. P. \& B. Dennis. 1993. Density dependence in rangeland grasshoppers (Orthoptera: Acrididae). Oecologia. 96:1-8.

Lavigne, R. J. \& R. E. Pfadt. 1966. Parasites and predators of Wyoming rangeland grasshoppers. University of Wyoming. Agricultural. Experiment Station Science Monographs. 3:4-31.

Marquis, R. J. \& C. J. Whelan. 1994. Insectivorous birds increase growth of white oak through consumption of leaf chewing insects. Ecology. 75:2007-2014.

Mitchel, J. E. \& R. E. Pfadt. 1974. A role of grasshoppers in a short grass prairie ecosystem. Environmental Entomology. 3:358-360.

Odum, E. P., C. E. Connell, \& L. P. Davenport. 1962. Population energy flow of the three primary consumer components of old field ecosystems. Ecology. 43:88-96.

Ovadia, O. \& O. J. Schmitz. 2004. Weather variation and trophic interactions strength: sorting the signal of the noise. Oecologia. 140:398-406.

Paine, R. T. 1966. Food web complexity and species diversity. The American Naturalist. 100: 65-73.

Pitt, W. C. 1999. Effects of multiple vertebrate predators on grasshopper habitat selection: trade-offs due to predation risk, foraging and thermoregulation. Evolutionary Ecology. 13: 499-515.

Polis, G. A. 1991. Complex trophic interactions in deserts: an empirical critique of food-web theory. The American Naturalist. 138:123-155.

Rees, N. E. 1973. Arthropod and nematode parasites, parasitoids and predators of Acrididae in America North of Mexico. U. S. Department of Agriculture Technical Bulletin. 460 pp. 
Richman, D.B., D.C. Lightfoot, C.A. Sutherland \& D.J. Ferguson. 1993. A Manual of the grasshoppers of New Mexico. Orthoptera: Acrididae and Romalidae. Handbook No. 7. Cooperative Extension Service New Mexico State University. U. S. 112 pp.

Risser, P. G., E.C. Birney, H. D. Blocker, S.W. May, W. J. Parton \& J. A. Wiens. 1981. The true prairie ecosystem. Hutchinson Ross, Pub. Co. Strodsberg, Philadelphia. U. S. 557 pp.

Rivera, E. 1986. Estudio faunístico de los Acridoidea de la Reserva de la Biosfera de Mapimí, Dgo. México. Acta Zoologica Mexicana (n. s.). 14:1-12.

Rivera G., E. 1988. Actividad diurna de Taeniopoda eques Burmeister (Orthoptera: Romaleidae), en el Bolsón de Mapimí, Dgo. México. Folia Entomologica Mexicana. 75:5-15.

Rivera G., E. 1992. Uso de microambientes por Trimerotropis pallidipennis (Burmeister), (Orthoptera: Acrididae) en un gradiente topográfico típico del Desierto Chihuahuense. Folia Entomologica Mexicana. 86:1-14.

Rivera G. E. 2006. An Annotated checklist of some orthopteroid insects of Mapimí Biosphere Reserve (Chihuahuan Desert), Mexico. Acta Zooogica. Mexicana (n. s.), 22: 131 -149.

Rota, J. \& D. L. Wagner. 2006. Predator Mimicry: Metalmark Moths Mimic Their Jumping Spider Predators. PLUS ONE 1(1): e45. dol:10.1371/journal.pone.0000045.

Rosenheim, J. A. 1998. Higher - order predators and the regulation of insect herbivore populations. Annual Review of Entomology 43:421-447.

Rothley, K. D., O. J. Schmitz, \& J. L. Cohen. 1997. Foraging to balance conflicting demands: novel insights from grasshoppers under predation risk. Behavioral Ecology. 8:551-559.

Schmitz, O. J. 1998. Direct and indirect effects of predation and predation risk in old-field interaction webs. The American Naturalist. 151:327-342.

Schmitz, O. J., A. P. Beckerman, \& K. M. O’Brien. 1997. Behaviorally-mediated trophic cascades: effects of predation risk on food web interactions. Ecology. 78: 1388-1399.

Schmitz, O. J., P. Hamback \& A. P. Beckerman. 2000. Trophic cascades in terrestrial systems: a review of the effect of top predators removal on plants. The American Naturalist. 155:141-153.

Staddon, J.E.R. \& R.P. Gendron. 1983. Optimal detection of cryptic prey may lead to predator switching. The American Naturalist. 122:843-848.

Steel, R. G. D. \& J. H. Torrie. 1960. Principles and procedures of statistics. Mc Graw Hill. Nueva York. 481 pp.

Steel, R. G. D. \& J. H. Torrie. 1976. Introduction to Statistics. Mc Graw Hill. New York. 382 pp.

Stower, W. J. \& D. F. Greathead. 1969. Numerical Changes in a population of the desert locust, with special reference to factors responsible for mortality. Journal of Applied Ecology. 6:203-235.

Van Hook, R. I. Jr. 1971. Energy and nutrient dynamics of spider and orthopteran populations in grassland ecosystem. Ecological. Monographs. 41:1-26.

Uvarov, B. 1977. Grasshoppers and Locusts: a handbook of general Acridology. Vol. 2. Behaviour, Ecology, Biogeography, population dynamics. Centre for Overseas Pest Research, Londres. England. 613 pp.

Wiens, J. A \& J. T. Rotenberry. 1979. Diet niche relationships among North American grassland and scrub steppe birds. Oecologia (Berlín), 42:453-492.

Zar, J. H. 1996. Biostatistical Analysis. Prentice Hall, New Jersey, U. S. 662 pp. 